\title{
Vectors of Crimean-Congo Hemorrhagic Fever (CCHF): Prevention and its Control
}

\author{
SN Sharma, Ram Singh, N Balakrishnan, Rina Kumawat, Sujeet Kumar Singh
}

National Centre for Disease Control Dte. General of Health Services, Ministry of Health \& FW, 22 - Sham Nath Marg, Delhi, India. DOI: https://doi.org/10.24321/0019.5138.202025

\section{I $\quad \mathbf{N} \quad \mathbf{F} \quad \mathbf{O}$}

\section{Corresponding Author:}

SN Sharma, National Centre for Disease Control Dte. General of Health Services, Ministry of Health \& FW, 22 - Sham Nath Marg, Delhi, India. E-mail Id:

drsns.nvbdcp@gmail.com

Orcid Id:

https://orcid.org/0000-0001-8569-1661

How to cite this article:

Sharma SN, Singh R, Balakrishnan N, Kumawat R, Singh SK. Vectors of Crimean-Congo Hemorrhagic Fever (CCHF): Prevention and its Control. J Commun Dis 2020; 52(3): 22-26.

Date of Submission: 2020-03-12

Date of Acceptance: 2020-05-05

\section{$\begin{array}{llllllll}\mathbf{A} & \mathbf{B} & \mathbf{S} & \mathbf{T} & \mathbf{R} & \mathbf{A} & \mathbf{C} & \mathbf{T}\end{array}$}

CCHF is caused by a tickborne virus (Nairovirus) in the family Bunyaviridae. It was first characterized in the Crimea in 1944 and given the name Crimean hemorrhagic fever. Later, it was recognized in 1969 as the cause of illness in the Congo, thus resulting in the current name of the disease. $\mathrm{CCHF}$ is a zoonotic viral disease that is asymptomatic in infected animals, but a serious threat to humans. The virus is mainly transmitted by tick species of the genus Hyalomma. During January 2011, Nosocomial (infections caught in hospitals) outbreak was detected in Gujarat, Ahmedabad. Thereafter, several outbreaks and cases of CCHF were reported in the states of Gujarat and Rajasthan during 2012-15. Cases were documented from six districts of Gujarat (Ahmedabad, Amreli, Patan, Surendranagar, Kutch and Aravalli), 3 districts of Rajasthan (Sirohi, Jodhpur and Jaisalmer) and in state of Uttar Pradesh were also affected. During 2019, 3 cases were reported in Rajasthan \& 17 cases reported from Gujarat from the districts of Bhavnagar, Botad, Amreli, Kheda, Jamnagar, Rajkot, Surendranagar, Morbi, Jodhpur. An attempt has been made to describe the vectors of CCHF in India and its prevention and control so as to benefit the program managers working at the grass root level.

Keywords: Crimean-Congo Hemorrhagic Fever (CCHF), Zoonotic, Hyalomma, Nosocomial, Ixodid, Bionomics

\section{Introduction}

$\mathrm{CCHF}$ is a zoonotic disease transmitted by ticks and characterized by fever and hemorrhage. ${ }^{1,2}$ It was first described in Soviet soldiers in the Crimea in 1944 and was named Crimean fever. In 1956, the virus was isolated from a child with similar symptoms and was named Congo virus. ${ }^{3}$ The causative agent of both illnesses was shown to be the same virus, which was subsequently termed CCHF virus. ${ }^{4}$ $\mathrm{CCHF}$ infects a range of animals; humans are the only known host that develops disease. CCHF is an emerging infectious disease given the expanding distribution of its main vector, ticks of the genus Hyalomma. Each year, more than 1000 human cases are reported from Europe and western Asia. ${ }^{5-7}$ The primary means of transmission to humans is via tick bites. The reservoir and vector of CCHFV are the ticks of the Hyalomma genus. Therefore, the circulation of this virus depends upon the distribution of the ticks. The virus can be transmitted from tick to animal, animal to human and human to human.

Vector, amplifying host and climatic conditions exist for centuries. Serological evidence of CCHF in camel, sheep and goats was reported by NIV Pune 2010 and Nosocomial infection reported in January 2011. CCHF outbreaks constitute a threat to public health, because 
of its epidemic potential, high case fatality, potential for nosocomial outbreaks and difficulties in treatment and prevention.

\section{Vectors of CCHF}

CCHFV is primarily transmitted via hard-bodied Hyalomma ticks of the family Ixodes, particularly Hyalomma marginatum. ${ }^{8}$ The geography of CCHF infection reflects the distribution of Hyalomma ticks, which have a northern geographic limit of $48^{\circ}$ north latitude. ${ }^{9} \mathrm{CCHFV}$ has also been isolated from Rhipicephalus, Boophilus and Dermacentor spp., which may also transmit the virus. ${ }^{10}$

Infected animals may also act as reservoir during the period of viremia. Most important source for acquisition of the virus by ticks is believed to be infected small vertebrates on which immature Hyalomma ticks feed. The larvae and nymphs mainly feed on the lower vertebrates such as rodents, rabbits and hares. Adults feed on higher vertebrates such as cattle, goat and sheep etc as well as man. Domestic ruminant animals, such as cattle, sheep and goats, are viraemic for around one week after becoming infected

Twenty-five tick species and sub-species have been reported to be CCHF virus reservoirs/vectors (the single record from an argasid, the bird parasitizing Argas persicus, remains to be confirmed). ${ }^{11}$ One-host ticks, Boophilus annulatus, $B$. microplus, $B$. decoloratus (and probably B. geigyi), appear to maintain intense virus inter-action for many weeks or months between several tick species infesting cattle. The 2-host vectors are Hyalomma m. marginatum, $H$. marginatum turanicum and $H$. marginatum rufipes (and probably $H$. marginatum isaaci); they feed as immatures on birds, hares, or hedgehogs and, as adults, chiefly on cattle. Other 2-host vectors, $H$. anatolicum, $H$. detritum, and Rhipicephalus bursa, feed both as immatures and adults on cattle. The $H$. marginatum complex, and $H$. a. anatolicum, are especially important in causing epidemics and outbreaks of human CCHF owing to their great numbers during certain periods and to their aggressiveness in seeking human hosts. Others, including 13 species of 3-host ticks [Haemaphysalis punctata, Amblyomma variegatum, Dermacentor (2 spp.), Hyalomma (5 spp.), and Rhipicephalus (4 spp.)], which generally seek human hosts less aggressively than the cited hyalommas, serve chiefly to maintain enzootic foci of CCHF virus circulation between ticks and wild and domestic mammals.

Ground-feeding birds are often hosts of CCHF virus-infected ticks, but birds apparently do not become viraemic. Unusually severe winter-spring weather, resulting in decimation of Hyalomma populations and also of hosts of immature stages, appears to have been largely responsible for virus circulation to revert from epizootic (epidemic) to enzootic intensity. Humans become infected, when bitten by infected ticks, or when crushing these ticks in their bare hands or shearing tick infested sheep. Household and nosocomial cases resulting from contamination by bloody discharges from CCHF patients have been especially numerous and severe, often with great mortality in villages and hospitals, where the disease was unrecognized.

\section{Ixodid (Hard Ticks)}

Ixodid (Hard) ticks are characterized by the presence of scutum (dorsal shield) and the capitulum (head) is terminal. Ticks are the obligate blood feeding arthropods from animals and man and all stages of life cycle viz., larvae, nymph and both sexes of adults are blood feeders. Ticks transmit a greater variety of infective agents, than any other group of haematophagous arthropods many of them are zoonotic diseases which are inter transmissible between animals and man. Toxins and most infectious agents are transmitted to vertebrate to salivary fluids inoculated while the parasite feeds.

\section{Genus; Hyalomma}

World: Mainly distributed from Africa, Asia and Europe, 30 known species. India: 9 species.

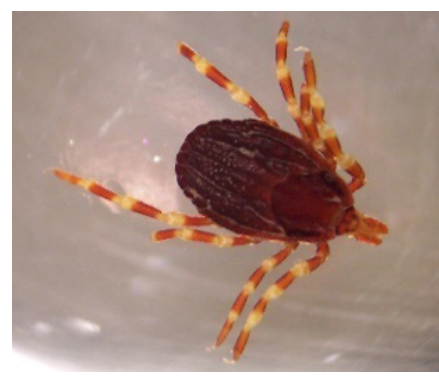

Figure I. Hylomma Tick

\section{Hyalomma Tick: Morphological Characters}

- Scant ornamentation sometimes bands present on legs (Figure 1).

- Eyes present, irregular festoons, partially consealed.

- Palpi long segment two approximately twice as long as segment 3.

- Basis capituli sub triangular dorsally.

- Coxa I deeply cleft. Males with adanal and accessory shields.

\section{Bionomics of Hyalomma Ticks}

Ticks of the genus Hyalomma remain on the same host during the larval and nymphal stages, the engorged nymph dropping to the soil where it moults to the adult form which then seeks a new host; ticks possessing this type of life cycle which involve two hosts are required are known as two-host ticks. The larvae and nymphs mainly feed on the lower vertebrates such as rodents, rabbits and hares while the adults feed on higher vertebrates such as cattle, goat and sheep etc as well as man. Trans-stadial and trans- 
ovarian route of transmission have been exhibited by the ticks and these phenomena are useful in the maintenance of tickborne arbo viruses in nature.

The members of the genus Hyalomma are extremely hard ticks, often existing under varied conditions of cold heat and aridity. $H$. anatolicum and $H$. marginatum are the vectors of CCHF. The engorged larvae and unfed adults of the $H$. anatolicum exhibit over wintering phenomenon by hibernating in cracks and wooden crevices in animal shelters of Russia and in rodent burrows in African deserts. The survival of larvae is up to 241 days, nymphs up to 246 days and adults over one year. H. marginatum often occurs in high numbers and is an aggressive human parasite and the unfed adults survive over 2 years. It may oviposit 4 to 15 thousand eggs in their lifetime.

\section{Reservoirs and Vectors of CCHF}

Hard ticks are the reservoir and vector of CCHF virus and in addition the infected animals may also act like a reservoir during the period of viremia. The CCHF virus may infect a wide range wild animal like hare, rodents and domestic animals such as sheep, goats, cattle and camel. Many birds are resistant to this infection except ostriches in endemic areas. Animals become infected with CCHF virus by the bite of infected ticks. The various environmental factors also influence the transmission of the virus. ${ }^{12,13}$

$\mathrm{CCHF}$ is transmitted mainly by tick vector Hyalomma anatolicum, and apart from this by $H$. marginatum, $H$. marginatum rufipes, $H$. marginatum turanicum and $H$. marginatum isaaci. Sporadic cases or import cases of CCHF virus (CCHFV) have been reported from Gujarat and Rajasthan recently during 2019. Antibody survey in domestic animals has shown a wide prevalence of $\mathrm{CCHF}$ throughout the country.

\section{Life Cycle}

CCHF vectors are two-host ticks and require two hosts to complete their life cycles. The adults lay eggs and emerging larvae attach to the vertebrate host. Hyalomma anatolicum parasitizes mainly cattle. The detachment and dropping rhythms of the species are so adjusted that these occur only at a time when the cattle is resting in the sheds normally at night time.

The engorged ticks that drop in the sheds find suitable niche in the cracks and the crevices, where the female ticks oviposit and the larvae and the nymph moult to the next stage. The questing larvae, the unfed nymphs and adults that have moulted from the previous stage can easily find their hosts in the cattle sheds. The humans acquire infection when they come in close contact of this environment.

A number of tick genera are capable of becoming infected with the CCHF virus, but the most efficient and common vectors for $\mathrm{CCHF}$ appear to be members of the Hyalomma genus. Trans-ovarial (transmission of the virus from infected female ticks to offspring via eggs) and venereal transmission have been demonstrated amongst some vector species, indicating one mechanism which may contribute to maintaining the circulation of the virus in nature.

\section{Vector Surveillance}

Sampling may be undertaken to identify tick habitats, where the cases have been reported and vector control may be needed. Collection of free-living ticks- unfed larvae, nymphs and adults by flagging/ drag net method (per man hour density to be recorded). Collection of ticks from domestic and wild animals to determine the tick index and isolation of virus is done from them. The frequency of sampling at monthly intervals and the frequency may be increased during epidemics. It is very difficult to select any site for vector surveillance to detect viral activity through ticks and the infestation rate of ticks among cattle (Sheep, Goat, Cows, Buffalos, Camel) due to their presence across the country with large population density. The ideal method may be to select collection sites based on the reporting of positive human CCHF cases and ecological parameters in within the affected area. Based on the total existing livestock in the affected area, an appropriate formula may derive to determine the sample size. The livestock may be investigated for tick collection during spring and summer seasons. ${ }^{14,15,16}$

\section{Surveillance Tools}

Active tick surveillance involves effective monitoring of prevalence, distribution, and infection rate among the vector ticks in a targeted geographical area. Efficiency of each method might vary based on the tick species, developmental stage, and host-seeking behavior. The following surveillance methods can be applied for the surveillance of CCHF vectors.

- Dragging, flagging, and dry ice-baited traps are the few collection methods, which targets hosts seeking tick population.

- Collection of tick parasitizing live host including rodent surveys.

- Leaf litter sampling method.

\section{Entomological Indicators}

The followings are the common entomological indicators being used for vector surveillance.

\section{Prevalence Rate of Hyalomma spp \\ Hyalomma tick species collected \\ Total number of ticks collected $\times 100$}

\section{Infestation Rate}

$\frac{\text { Total positive livestock for ticks }}{\text { Total livestock surveyed or investigated }} \times 100$ 


\section{Tick Index}

$$
\frac{\text { No. of ticks collected }}{\text { Total Numbers of Hosts examined }} \times 100
$$

\section{RNA Extraction and CCHFV Genome Detection}

Ticks collected from each host are kept alive in separate labelled tubes, and then processed for identification by morphological characteristics using a stereomicroscope on the basis of valid identification keys. Identified ticks were kept in micro tubes for testing with both an antigendetecting Enzyme-Linked Immunosorbent Assay (ELISA) and RT real-time PCR (RT PCR) method. Ticks species identified and processed from each host is considered as a single pool.

\section{Virus Detection Rate}

No. of Ticks found positive for CCHF Virus / No. of ticks processed for CCHF virus detection

\section{Prevention \& Control}

In the areas, endemic for tickborne diseases like CCHF the integrated tick control strategy need to be undertaken which has the following components.

\section{Surveillance}

Sampling to identify tick habitats where control is needed. Collection of free-living ticks- unfed larvae, nymphs and adults by flagging/ drag net method (Per man hour density to be recorded). Collection of ticks has to be carried out from domestic and wild animals to determine the tick index and isolation of virus from them. The frequency of sampling at monthly intervals and the frequency may be increased during epidemics.

\section{Vegetation Management}

Physical or chemical measures to reduce and isolate tick habitats. In areas with tick infestation like camp sites and parks, ticks can be controlled by removal of vegetation serving as their habitat by cutting, mowing or by applying herbicides.

\section{Host Management}

This is practice by the removal or exclusion of host animals: Tick populations can be controlled by removing of animals on which they usually feed. Fences may also be made to exclude wild animals from entering into human habitation.

\section{Targeted Chemical Control}

Pesticide applications need to be under taken against ticks, at the tick host or habitat. In areas with tick infestation they can be controlled by insecticidal application mainly by applying bio degradable insecticidal dusts. The animal houses, houses, furniture's and wall crevices and cavities should be treated with residual insecticides. The heavy tick infested animals also treated with insecticidal dusts and formulation on their body. Spraying should be undertaken in the forest tracts frequented by the villagers. Surveillance and monitoring of ticks' infestation on animals and occurrence of human cases in a locality on long term basis.

Various insecticidal formulations can apply to domestic pets, such as dogs, to get rid of their ticks. Recommended treatment includes solution of $0.5 \%$ Dichlorvos (DDVP), 1\% Carbaryl (Sevin) or 3-5\% Malathion can be applied to the coats of animal habitats. The dipping of sheep and cattle, and sometime other livestock, in acaricidal bathes, or spraying them with insecticides. It is crucial if ticks and tickborne diseases of man as well as livestock rate to be effectively controlled.

\section{Cultural Practices}

Lifestyle changes to limit the exposure of ticks. Avoiding of sitting and lying down on tick infested ground. IEC need to be imparted to the public on the ticks and ticks borne diseases. Vector control measures in the forest peripheral and high-risk villages to be carried out. Entomological, epidemiological and serological surveillance should be undertaken in new areas bordering the affected districts.

\section{Personal Protection}

Protective clothing repellents checking for and removing of ticks. Personal protection form tick bites are mainly by use of protective clothing (full length clothing). The persons return from tick infested areas should examine the body for tick infestation and their removal through scrubbing. Washing of clothes and body with hot water and soap need to be undertaken after visiting the forest to get rid of tick infestation. Vaccinating the people at risk and persons looking after animals.

\section{Conclusion}

Studies can be undertaken to undertake vector surveillance for $\mathrm{CCHF}$ to understand the prevalence in different ecological zones and associated hosts. Virus isolation can also be undertaken among some pools of vector species to understand the dynamics of CCHF transmission. Thereafter, a comprehensive plan of action can be made for the prevention and control of the diseases.

\section{Conflicts of Interest: None}

\section{References}

1. van Eeden PJ, Joubert JR, van de Wal BW et al. A nosocomial outbreak of Crimean-Congo haemorrhagic fever at Tygerberg Hospital. Part I. Clinical features. $S$ Afr Med J 1985; 68: 711.

2. Whitehouse CA. Crimean-Congo hemorrhagic fever. Antiviral Res 2004; 64: 145.

3. Hoogstraal H. The epidemiology of tick-borne CrimeanCongo hemorrhagic fever in Asia, Europe, and Africa. 
J Med Entomol 1979; 15: 307.

4. Simpson DI, Knight EM, Courtois G et al. Congo virus: a hitherto undescribed virus occurring in Africa. I. Human isolations-clinical notes. East Afr Med J 1967; 44: 86.

5. Mertens M, Schmidt K, Ozkul A, et al. The impact of Crimean-Congo hemorrhagic fever virus on public health. Antiviral Res 2013; 98: 248.

6. Maltezou HC, Papa A. Crimean-Congo hemorrhagic fever: risk for emergence of new endemic foci in Europe? Travel Med Infect Dis 2010; 8: 139.

7. Leblebicioglu H, Ozaras R, Sunbul M. Crimean-Congo hemorrhagic fever: A neglected infectious disease with potential nosocomial infection threat. Am J Infect Control 2017.

8. Anagnostou V, Papa A. Evolution of Crimean-Congo hemorrhagic fever virus. Infect Genet Evol 2009; 9: 948.

9. Papa A, Dalla V, Papadimitriou E et al. Emergence of Crimean-Congo haemorrhagic fever in Greece. Clin Microbiol Infect 2010; 16: 843.

10. Hoogstraal $\mathrm{H}$. Changing patterns of tickborne diseases in modern society. Annu Rev Entomol 1981; 26: 75.

11. Hoogstraal $\mathrm{H}$. The epidemiology of tick-borne CrimeanCongo hemorrhagic fever in Asia, Europe, and Africa. Journal of Medical Entomology 1979; 15(4): 307-417.

12. Geevarghese G, Dhanda V. The Indian Hyalomma ticks. Indian Council of Agricultural Research, Krishi Anusandhana Bhavan, New Delhi. 1987.

13. Shanmugam J, Smirnova SE, Chumakov MP. Presence of antibodies to arboviruses of the Crimean haemorrhagic fever Congo (CHF-Congo) group in human being and domestic animals in India. Indian J Med Res 1976; 64: 1403-13.

14. Mishra AC, Mehta M, Mourya DT, et al. Crimean-Congo haemorrhagic fever in India. Lancet 2011; 378: 372.

15. Rodrigues FM, Padbidri VS, Ghalsasi GR, et al. Prevalence of Crimean haemorrhagic-Congo virus in Jammu and Kashmir State. Indian J Med Res 1986; 84: 134-138.

16. Balakrishnan N. Current scenario of tickborne diseases in India: A review. J Commun Dis 2017; 14(2): 6-13. 